\title{
CONDITIONAL EXPECTATION IN AN OPERATOR ALGEBRA, III
}

\author{
By HisahaRU UMegaki
}

\section{Introduction.}

The theory of rings of operators of von Neumann has been developed by many authors, especially since it has been regarded as a non-commutative extension of the integration over a measure space by Dixmier [3], ${ }^{1)}$ Dye [4] and Segal [10], some fundamental theorems on measure theory have been extended. In the papers [3], [8] and [14], the authors have introduced the concept of the conditional expectation into some ring of operators of von Neumann (=von Neumann algebra in the sense of Dixmier [2] and we shall use this terminology below) which can be also regarded as a non-commutative extension of conditional expectation in the probability theory. The extension has also been made of the C. Moy characterization theorem (cf. [8] and [14]) and the martingale convergence theorem (cf. [15]). In the present note, as a part of a non-commutative extension of measure theoretic probability theory we shall prove for a von Neumann algebra of finite class a Halmos-Savage theorem (cf. [5]) with respect to sufficient statistics in probability theory which was reformulated under the terminology of Borel subfield by Bahadur [1].

For our purpose we shall depend upon as a basic theorem the RadonNikodym theorem due to Dye [4], in a von Neumann algebra. Firstly we shall introduce a space of some restricted normal states relative to a von Neumann subalgebra which will be called tracelet space (cf. Definition 1) and give an example of such space by the direct product of finite factors in the sense of Nakamura [7], and further we shall extend the existence theorem of the conditional expectation in a von Neumann algebra for normal states in the tracelet space (cf. Theorem 1). Under Theorem 1, we shall introduce a concept of sufficiency of von Neumann subalgebra for some restricted set of normal states and prove a Halmos-Savage characterizations of sufficient subalgebra (cf. Theorems 5 and 6). These have applications to find a characteristic property of subalgebra having unique expectation onto it (cf. Theorem 7 ), and to prove a von Neumann proposition (cf. Theorem 2 of [9]) for a von Neumann's operations which are stated in the final section.

\section{Preliminary.}

Let $A$ be a countably decomposable (= $\sigma$-finite) von Neumann algebra of

Received November 27, 1958.

1) The numbers in brakects refer to the reference at the end of this paper. 
finite class acting on a Hilbert space $H$, and $B, C$ and $M$ von Neumann subalgebras of $A$, and $B^{c}=B^{\prime} \frown A$ where $B^{\prime}$ is the commutant of $B$. Denote the identity operator by 1 and a complex number by $\lambda$.

Let $\mu$ be arbitray but fixed faithful normal trace of $A$. Then for a subalgebra $B$, there exists a linear mapping $e\left(a \rightarrow a^{e}\right)$ from $A$ onto $B$ such that for any $a \in A$ and for any $b \in B$

(1) $\mu(a b)=\mu\left(a^{e} b\right)$,

(2) $a^{e}=0(a \geqq 0)$ implies $a=0$,

(3) $a^{e} \geqq 0(a \geqq 0), a^{* e}=a^{e *}$ and $(a b)^{e}=a^{e} b$,

(4) $a_{\alpha}{ }^{e} \uparrow a^{e}$ if $a_{\alpha} \uparrow a$.

(cf. [3], [8] and [14]). Such a linear mapping $e$ is uniquely determined within the condition (1), that is, uniquely determined by $\mu$ and $B$, and it was called conditional expectation relative to $B$ (cf. [14]) or expectation conditioned by $B$ (cf. [8]). (Recently, Tomiyama [13] has proved that every linear idempotent mapping from $A$ onto $B$ with norm one has always the property (3)). Here we shall call a linear idempotent normal (=condition (4)) mapping from $A$ onto $B$ by $B$-expectation.

For the normal trace $\mu$, denote $L^{1}(A, \mu)\left(=L_{\mu}{ }^{1}\right.$ say) and $L^{2}(A, \mu)\left(=L_{\mu}{ }^{2}\right.$ say) the $L^{1}$ and $L^{2}$ spaces with the norms \|\|$_{1}$ and \|\|$_{2}$ respectively, and similarily denote for the subalgebra $B L^{1}(B, \mu)$ and $L^{2}(B, \mu)$ which are considered as closed subspaces of $L_{\mu}{ }^{1}$ and $L_{\mu}{ }^{2}$ respectively. Then the conditional expectation $e$ relative to $B$ is uniquely extended to a positive linear idempotent mapping from $L_{\mu}{ }^{1}$ onto $L^{1}(B, \mu)$ satisfying $\left\|x^{e}\right\|_{1} \leqq\|x\|_{1}$ for every $x \in L_{\mu}{ }^{1}$, and furthermore $e$ extended to a projection operator from $L_{\mu}{ }^{2}$ onto $L^{2}(B, \mu)$. We denote also the extended mappings of $e$ to $L_{\mu}{ }^{1}$ and $L_{\mu}{ }^{2}$ respectively by same $e$.

If a measurable operator $x$ (in the sense of Segal [10]) satisfies $x \eta B$ (i.e. $u x=x u$ for every unitary $u$ in $B^{\prime}$ ) then $x$ is called $B$-measurable.

For a normal state $\sigma$, a positive measurable operator $x$ is called $\sigma$-integr$a b l e$ if for the spectral resolution $x=\int \lambda d E_{\lambda}$ the numerical integration $\int \lambda d \sigma\left(E_{\lambda}\right)$ is finite, and we denote it by $\sigma(x)$. A measurable operator $x$ is $\sigma$-integrable if the absolute value $|x|$ of $x$ is $\sigma$-integrable. For any pair of measurable operators $x$ and $y$, if $|x-y|$ is $\sigma$-integrable and $\sigma(|x-y|)=0$, then we call “ $x=y$-n.e.". Denote $L^{2}(A, \sigma)$ the space of all measurable operators $x$ such that $\sigma\left(x^{*} x\right)$ is finite (i.e. $x^{*} x$ is $\sigma$-integrable). For $x$ and $y \in L^{2}(A, \sigma), y^{*} x$ is $\sigma$-integrable, and the space $L^{2}(A, \sigma)$ is a Hilbert space under the inner product $\langle x, y\rangle_{\sigma}=\sigma\left(y^{*} x\right)$ for $x, y \in L^{2}(A, \sigma)$ where the origin 0 in $L^{2}(A, \sigma)$ being $\sigma$-n.e. zero operator.

For any pair of normal states $\sigma_{1}, \sigma_{2}$, we call $\sigma_{1}$ being absolutely continuous with respect to $\sigma_{2}$ (denote $\left.\sigma_{1} \prec \sigma_{2}\right)$, if $\sigma_{2}(a)=0(a \geqq 0)$ implies always $\sigma_{1}(a)=0$. Further for any pair of sets of normal states $S_{1}$ and $S_{2}$, if $\sigma_{2}(a)=0(a \geqq 0)$ for every $\sigma_{2} \in S_{2}$ implies $\sigma_{1}(a)=0$ for every $\sigma_{1} \in S_{1}$, we denote ' $S_{1} \prec S_{2}$ ', and if $S_{1} \prec S_{2}$ and $S_{2} \prec S_{1}$, denote ' $S_{1} \sim S_{2}$ '. If $\rho \prec \sigma$, by Radon- 
Nikodym theorem due to Dye [4] there exists uniquely an operator $d \in L^{2}(A, \sigma)$ within $\rho$-n.e. such that $\rho(a)=\sigma\left(d^{*} a d\right)$ for every $a \in A$, and we denote $D(\rho / \sigma)=d$ and call the Radon-Nikodym derivative in the sense of Dye (of $\rho$ with respect to $\sigma$ ).

If $S$ is a set of measurable operators, then denote the set of all positive operators in $S$ by $S^{+}$.

Denote " $s_{\sigma}$ " the supporting projection of a normal state $\sigma$, and for a von Neumann subalgebra $B$ " $b_{\sigma}$ " the supporting projection in $B$ of the $\sigma$. For any von Neumann algebra $M$, denote the Banach space of all $\sigma$-weakly continuous linear functionals of $M$ by $M_{*}$ in the notation of [3].

\section{Tracelet space and correspondence between normal states and ex-} pectations.

In the present section, firstly we shall introduce a some restricted set of normal states of $A$ which will be referred throughout of this paper.

Definition 1. For a von Neumann subalgebra $B$, let " $S_{B}$ " be the set of all normal states $\sigma$ of $A$ satisfying

$$
\sigma(a b)=\sigma(b a) \text { for every } a \in A \text { and for every } b \in B,
$$

and we call $S_{B}$ the B-tracelet space.

The $B$-tracelet space contains obviously the space of all normal traces, and is weakly closed, convex set in $A_{*}$. The following lemma follows immediately from the definition of $B$-tracelet space and the Radon-Nikodym theorem due to Dye:

Lemma 1. For any $\sigma \in S_{B}$, there exists $d_{\sigma} \in L^{1}\left(B^{c}, \mu\right)^{+}$such that $\sigma(a)=\mu\left(d_{\sigma} a\right)$ for every $a \in A$. Further the derivative $D(\sigma / \mu)$ is self-adjoint, positive and $d_{\sigma}=D(\sigma / \mu)^{2}$. Conversely for any $t \in L^{1}\left(B^{c}, \mu\right)^{+}$with $\|t\|_{1}=1$ $\sigma(a)(=\mu(t a))$ belongs to $S_{B}$.

We shall now give an example of $B$-tracelet space:

ExAmple $1 .^{2)}$ If $A$ is a $\mathrm{II}_{1}$-factor and $B, C$ are subfactors of $A$ such that $A=B \otimes C$ in the sense of Nakamura [7], where $B$ and $C$ are identifying with $B \otimes\{\lambda 1\}$ and $\{\lambda 1\} \otimes C$ respectively. Then obviously $B^{c}=C$ and $B=C^{c}$. Let $\mu_{B}$ and $\mu_{C}$ be the normal traces of $B$ and $C$ respectively, and $S(B)$ and $S(C)$ the sets of all normal states of $B$ and $C$ respectively. Then $\mu=\mu_{B} \times \mu_{C}$

2) This example is suggested by Prof. M. Nakamura. Takesaki [12] has extended the theorem of Nakamura [7] to the direct product of factors without the assumption of finiteness. If we introduce formally the tracelet space into a von Neumann algebra, without assuming the finiteness, in the same way of Definition 1 , then, when $B$ is $\mathrm{II}_{1}$ factor, $C$ is a factor and $A=B \otimes C$ in the sense of [12], the $B$-tracelet space $S_{B}$ is given by (6), where $\mu_{B}$ is the trace of $B$, and where $B$ and $B \otimes\{\lambda 1\}$ are identifying as in this Example. 
by $[7]$, and

$$
\begin{aligned}
& S_{B}=\left\{\mu_{B} \times \rho_{C} ; \rho_{C} \in S(C)\right\} \\
& S_{C}=\left\{\sigma_{B} \times \mu_{C} ; \sigma_{B} \in S(B)\right\}
\end{aligned}
$$

where $\mu_{B} \times \rho_{C}$ is defined as a state of $A$ by

$$
\left(\mu_{B} \times \rho_{C}\right)\left(\sum b_{i} \otimes c_{i}\right)=\sum \mu_{B}\left(b_{i}\right) \rho_{C}\left(c_{i}\right)
$$

for any finite $b_{i} \in B, c_{i} \in C(i=1,2, \cdots, n)$, that is, $B$ - or $C$-tracelet spaces are represented by $(6)$ or $\left(6^{\prime}\right)$ respectively. We shall prove (6): the state $\mu_{B} \times \rho_{C}$ obviously satisfies (5) and belongs to $S_{B}$. Conversely, let $\sigma \in S_{B}$ and let $\sigma_{C}$ be the state of $C$ of the contraction of $\sigma$ onto $C$. By Lemma 1 there is $d_{\sigma} \in L^{1}(C, \mu)^{+}$such that $\sigma_{C}(c)=\mu_{C}\left(d_{\sigma} c\right)$ for every $c \in C$. Let $\left\{c_{n}\right\}$ be a sequence of operators in $C$ satisfying $\left\|d_{\sigma}-c_{n}\right\|_{1} \rightarrow 0$. Then

$$
\begin{aligned}
\sigma(b \otimes c) & =\lim _{n \rightarrow \infty} \mu\left(\left(1 \otimes c_{n}\right)(b \otimes c)\right)=\lim _{n \rightarrow \infty} \mu\left(b \otimes c_{n} c\right) \\
& =\mu_{B}(b) \lim _{n \rightarrow \infty} \mu_{C}\left(c_{n} c\right)=\mu_{B}(b) \mu_{C}\left(d_{\sigma} c\right)=\mu_{B}(b) \sigma_{C}(c),
\end{aligned}
$$

and we get (6).

In the following, for any fixed von Neumann subalgebra $B$ of $A$ we shall give a correspondence between normal states in $S_{B}$ and $B$-expectations which is a generalization of the existence theorem of conditional expectation in $A$ (cf. Theorem 1 of [14]).

THeOREm 1. For any $\sigma \in S_{B}$, there corresponds a B-expectation $\varepsilon_{\sigma}$ such that

$$
\sigma(a)=\sigma\left(a^{\varepsilon_{\sigma}}\right) \quad \text { for every } a \in A,
$$

and $\varepsilon_{\sigma}$ is uniquely determined by $\sigma$ within the equation (7) and $\sigma-n . e$. Conversely any $B$-expectation $\varepsilon$ is corresponding to certain $\sigma \in S_{B}$, that is, $\varepsilon=\varepsilon_{\sigma}{ }^{3)}$.

Proof. Putting $f_{a}(b)=\sigma(a b)$ for any fixed $a \in A$ and for any $b \in B, f_{a}$ is a weakly* ( $=\sigma$-weakly) continuous linear functional of $B$. For each $b \in B$, let $u$ being the partially isometric operator in $B$ such that $b=u|b|$,

$$
\begin{aligned}
\left|f_{a}(b)\right| & =|\sigma(a b)|=|\sigma(a u|b|)|=\left|\sigma\left(a u b_{1} b_{1}\right)\right| \quad \quad\left(b_{1}=|b|^{1 / 2}\right) \\
& \left.=\left|\sigma\left(b_{1} a u b_{1}\right)\right| \leqq\|a u\|_{\infty} \sigma\left(b_{1} b_{1}\right) \leqq\|a\|_{\infty} \sigma(|b|) .^{4}\right)
\end{aligned}
$$

Hence there exists uniquely $a^{\prime} \in B$ such that $f_{a}(b)=\sigma\left(a^{\prime} b\right)$ and $b_{\sigma} a^{\prime}=a^{\prime}$ where $b_{\sigma}$ is the supporting projection in $B$ of $\sigma$ which belongs to the center of $B$. This implies $\sigma(a b)=\sigma\left(a^{\prime} b\right)$ for every $a \in A$ and every $b \in B$. The mapping $a \rightarrow a^{\prime}$ obtained above maps linearly $A$ into $B$ and satisfies (3) and (4). Indeed, for any $a, a_{1}, a_{2} \in A$ and for any $b \in B$

3) In general, for the $B$-expectation, there corresponds a number of states in the $B$-tracelet space $S_{B}$. Concerning this fact we shall discuss the sufficiency of subalgebra for a set of normal states in $S_{B}$.

4) $\|a\|_{\infty}$ is the operator bound of $a$. 


$$
\begin{aligned}
\sigma\left(\left(a_{1}+a_{2}\right)^{\prime} b\right) & =\sigma\left(\left(a_{1}+a_{2}\right) b\right)=\sigma\left(a_{1} b\right)+\sigma\left(a_{2} b\right) \\
& =\sigma\left(a^{\prime} b\right)+\sigma\left(a^{\prime} b\right)=\sigma\left(\left(a^{\prime}+a^{\prime}\right) b\right) .
\end{aligned}
$$

Since $a_{1}{ }^{\prime}, a_{2}{ }^{\prime}$ and $\left(a_{1}+a_{2}\right)^{\prime}$ belong to $b_{\sigma} B,\left(a_{1}+a_{2}\right)^{\prime}=a_{1}{ }^{\prime}+a_{2}{ }^{\prime}$. Similarily $(\lambda a)^{\prime}=\lambda a^{\prime}$ and the condition (3) is obviously satisfied. The condition of normality (4) for the mapping $a \rightarrow a^{\prime}$ follows from that: If $a_{\alpha} \uparrow a$ for self-adjoint $a_{\alpha}, a \in A$, then for $b \in B^{+}$

$$
\sigma\left(a_{\alpha}^{\prime} b\right)=\sigma\left(a_{\alpha} b\right) \uparrow \sigma(a b)=\sigma\left(a^{\prime} b\right)
$$

and $a_{\alpha^{\prime}} \leqq a^{\prime}$ imply $a_{\alpha^{\prime}} \uparrow b_{0}$ for some self-adjoint $b_{0} \in b_{\dot{\sigma}} B$. Hence $\sigma\left(b_{0} b\right)=\sigma\left(a^{\prime} b\right)$ for all $b \in B$ and $a^{\prime}=b_{0}$. Now we put

$$
a^{\varepsilon_{\sigma}}=a^{\prime}+a^{e}\left(1-b_{\sigma}\right) \quad \text { for every } a \in A,
$$

where $a^{e}$ is the conditional expactaion of $a$ relative to $B$, then for every $b \in B$

$$
b^{\varepsilon_{\sigma}}=b^{\prime}+b^{e}\left(1-b_{\sigma}\right)=b b_{\sigma}+b\left(1-b_{\sigma}\right)=b
$$

and $A^{\varepsilon_{\sigma}}=B$. Therefore $\varepsilon_{\sigma}$ is obviously a $B$-expectation and satisfies (7). The uniquess of $\varepsilon_{\sigma}$ : Taking a $B$-expectation $\varepsilon$ satisfying $\sigma(a)=\sigma\left(a^{\varepsilon}\right)$ for all $a \in A$,

$$
\sigma\left(a^{\varepsilon} b\right)=\sigma\left((a b)^{\varepsilon}\right)=\sigma(a b)=\sigma\left((a b)^{\varepsilon} \sigma\right)=\sigma\left(a^{\varepsilon} \sigma b\right)
$$

for every $b \in B$. (8) and $s_{\sigma} \leqq b_{\sigma}$ imply that $b_{\sigma} a^{\varepsilon_{\sigma}}=b_{\sigma} a^{\varepsilon}$ and $s_{\sigma} a^{\varepsilon}=s_{\sigma} b_{\sigma} a^{\varepsilon}=s_{\sigma} b_{\sigma} a^{\varepsilon_{\sigma}}$ $=s_{\sigma} a^{\varepsilon} \sigma$, and we obtain $a^{\varepsilon}=a^{\varepsilon_{\sigma}} \sigma-n . e$.

Conversely, let $\varepsilon$ be arbitary $B$-expectation. Putting $\sigma(a)=\mu\left(a^{\varepsilon}\right), \sigma\left(a^{\varepsilon}\right)$ $=\mu\left(a^{\varepsilon} \sigma\right)=\mu\left(a^{\varepsilon}\right)=\sigma(a)$ and

$$
\sigma(a b)=\mu\left((a b)^{\varepsilon}\right)=\mu\left(a^{\varepsilon} b\right)=\mu\left(b a^{\varepsilon}\right)=\mu\left((b a)^{\varepsilon}\right)=\sigma(b a) .
$$

These imply that $\sigma \in S_{B}$ and $a^{\varepsilon}=a^{\varepsilon_{\sigma}} \sigma-n . e .$.

REMARK 1. The $B$-expectation $\varepsilon_{\sigma}$ corresponding to $\sigma \in S_{B}$ is uniquely determined by $\sigma$ within (7) and without the terminology $\sigma$-n.e. if and only if $\sigma$ is faithful on $B$, because the both parts of "if" and "only if" follow from that the support $b_{\sigma}$ in $B$ of $\sigma$ is identity operator 1 . In Theorem 1, even if the assumption on $A$-the countable decomposability and the finiteness - does not put, and if the set of states $S_{B}$ defined by (5) is not empty, then for any $\sigma \in S_{B}$ there corresponds uniquely a linear idempotent mapping from $A$ onto $b_{\sigma} B$ satisfying (3) and (4). This fact will be obtained by the same way as in the proof of Theorem 1 .

REMARK 2. As Example 1, let $A, B$ and $C$ be $\mathrm{II}_{1}$-factors such that $A=B \otimes C$, and let $\mu, \mu_{B}$ and $\mu_{C}$ be the normal traces of $A, B$ and $C$ respectively. Then by Example 1 any $\sigma \in S_{B}$ is expressed by $\sigma=\mu_{B} \times \sigma_{C}$ where $\sigma_{C}$ is the contraction of $\sigma$ onto $C$ (i.e. $\sigma_{C}(c)=\sigma(1 \otimes c)$ ). This implies that for any finite $b_{i} \in B$ and $c_{i} \in C$

$$
\left(\sum_{i} b_{i} \otimes c_{i}\right)^{\varepsilon_{\sigma}}=\sum_{i} b_{i} \otimes \sigma_{C}\left(c_{i}\right) 1
$$

Indeed, for any $b \in B$ 


$$
\begin{aligned}
\sigma\left(\left(\sum b_{i} \otimes \sigma_{C}\left(c_{2}\right) 1\right)(b \otimes 1)\right) & =\sigma\left(\sum b_{i} b \otimes \sigma_{C}\left(c_{i}\right) 1\right) \\
=\Sigma\left(\mu_{B}\left(b_{i} b\right) \sigma_{C}\left(c_{i}\right)\right) & =\sigma\left(\left(\sum b_{i} b \otimes c_{i}\right)\right)=\sigma\left(\left(\sum b_{i} \otimes c_{\imath}\right)(b \otimes 1)\right)
\end{aligned}
$$

and hence (9) holds.

In the following we prove that any $B$-expectation is representable by the conditional $B$-expectation $e$ as (10) below.

THEOREM 2. For any B-expectation $\varepsilon$, there exists uniquely $t_{\varepsilon} \in L^{1}\left(B^{c}, \mu\right)^{+}$ such that

$$
a^{\varepsilon}=\left(t_{\varepsilon} a\right)^{e} \quad \text { for every } a \in A .
$$

Conversely, for any $t \in L^{1}\left(B^{c}, \mu\right)^{+}$with $t^{e}=1$, putting $a^{\varepsilon}=(t a)^{e}$, then $\varepsilon$ is a $B$-expectation. The correspondence $\varepsilon \rightarrow t_{\varepsilon}$ is one-to-one between the set of all $B$-expectations and $\left\{t \in L^{1}\left(B^{c}, \mu\right)^{+} ; t^{e}=1\right\}$.

Proof. Putting $\sigma(a)=\mu\left(a^{\varepsilon}\right)$, by the proof of Theorem $1 \sigma \in S_{B}$. Then by Lemma 1 there exists uniquely $d_{\sigma} \in L^{1}\left(B^{c}, \mu\right)^{+}$such that $\sigma(a)=\mu\left(d_{\sigma} a\right)$ for for all $a \in A$. Therefore for every $b \in B$

$$
\mu\left(a^{\varepsilon} b\right)=\mu\left((a b)^{\varepsilon}\right)=\sigma(a b)=\mu\left(d_{\sigma} a b\right)=\mu\left(\left(d_{\sigma} x\right)^{e} b\right)
$$

and $a^{\varepsilon}=\left(d_{\sigma} a\right)^{e}$. The operator $d_{\sigma}$ is the required one, i.e. $t_{\varepsilon}=d_{\sigma}$. The uniqueness will be followed from the final part in this proof.

Converse case: For any $t_{1} \in L_{\mu}^{2}, t_{2} \in L^{2}\left(B^{c}, \mu\right)$ and $b \in B$,

$$
\mu\left(\left(t_{1} t_{2}\right)^{e} b\right)=\mu\left(t_{1} t_{2} b\right)=\mu\left(t_{1} b t_{2}\right)=\mu\left(t_{2} t_{1} b\right)=\mu\left(\left(t_{2} t_{1}\right)^{e} b\right)^{5)}
$$

and $\left(t_{1} t_{2}\right)^{e}=\left(t_{2} t_{1}\right)^{e}$. Let $t \in L^{1}\left(B^{c}, \mu\right)^{+}$be $t^{e}=1$ and put $a^{\varepsilon}=(t a)^{e}$. Then $b^{\varepsilon}=(t b)^{e}=t^{e} b=b$ for any $b \in B$ and $(t a)^{e}=\left(t^{1 / 2} a t^{1 / 2}\right)^{e} \geqq 0$ for any $a \in A^{+}$. Hence for $a \in A^{+} 0 \leqq a^{\varepsilon}=(t a)^{e} \leqq\left(t\|a\|_{\infty} 1\right)^{e}=\|a\|_{\infty} 1$, and $a^{\varepsilon} \in B^{+}$. By the linearity of $\varepsilon, a^{\varepsilon} \in B$ for all $a \in A$. Since the normality of $\varepsilon$ is obvious, $\varepsilon$ is a $B$ expectation.

The final part follows immediately from the above parts and the following: If $t_{1}, t_{2} \in L^{\prime}$ satisfy $\left(t_{1} a\right)^{e}=\left(t_{2} a\right)^{e}$ for every $a \in A$, then $\mu\left(t_{1} a\right)=\mu\left(\left(t_{1} a\right)^{e}\right)$ $=\mu\left(\left(t_{2} a\right)^{e}\right)=\mu\left(t_{2} a\right)$ and $t_{1}=t_{2}$.

\section{Sufficiency of subalgebra.}

In this section, we shall introduce the concept of sufficient statistics of probability into the von Neumann algebra $A$.

Definition 2. Let $B$ be a von Neumann subalgebra and $S_{0}$ be a set of normal states of $A$. Then we call that $B$ is sufficient for $S_{0}$, if the $B$-tracelet space contains $S_{0}$ (i.e. $S_{0} \subset S_{B}$ ) and for each $a \in A$ there exists $a^{\prime} \in B$ such that $a^{\varepsilon_{\sigma}}=a^{\prime} \sigma-n . e$. for every $\sigma \in S_{0}$.

5) For any measurable operators $t$ and $t^{\prime}, t t^{\prime}$ is the closure of the product operator, i.e., $t t^{\prime}=t \cdot t^{\prime}$ in the notation of $[10]$. 
This definition is slightly non-commutative extension of the case of sufficient statistics in probability theory (cf. [1] and [5]), which may be described as the following:

Let $(\Omega, \mathfrak{Z})$ be a measurable space in the terminology of measure theory, and $\boldsymbol{A}$ the multiplication algebra of all bounded, measurable and complex valued functions defined on $\Omega$. Let $\mathfrak{B}$ be an arbitary Borel subfield of $\mathfrak{A}$ and $\boldsymbol{B}$ the subalgebra of $\boldsymbol{A}$ consisting of all $\mathfrak{B}$-measurable functions. Then $\boldsymbol{A}$ is (in general homomorphically) represented by a commutative von Neumann algebra $A$ acting on a Hilbert space $H^{6)}$ and $\boldsymbol{B}$ is similarily represented by a von Neumann subalgebra $B$ of $A$. The space of all probability measures of $(\Omega, \mathfrak{A})$ can be identified with the space of all normal states of $A$ by the natural way, which coincides with the $B$-tracelet space (likewise defined by (5) in Definition 1), because $A$ is commutative. We denote the element in $\boldsymbol{A}$ and its corresponding operator in $A$ by the same symbol $a$, etc.. Then for each probability measure $\sigma$, there corresponds a conditional expectation $E_{\sigma}(\mathfrak{g} \mid \mathfrak{B})(\mathfrak{g} \in \mathfrak{Z})$ relative to $\mathfrak{B}$ which is determined in $\sigma-n . e$. in the usual sense. In our notation, it is expressed by $E_{\sigma}(a \mid \mathfrak{B})=a^{\varepsilon_{\sigma}}$ for $a \in A$ (cf. Theorem 1 and Remark 1). For a set $S_{0}$ of probability measures, the sufficiency of a Borel subfield $\mathfrak{B}$ is defined by such a way: for any subset $\mathfrak{g} \in \mathfrak{A}$ there is a function $a^{\prime} \in B$ such that

$$
E_{\sigma}(\mathfrak{g} \mid \mathfrak{B})=a^{\prime} \quad \sigma \text {-n.e. } \quad \text { for every } \sigma \in S_{0} .
$$

For these cases the countable decomposability is not assumed, but since the equation (11) is defined within the " $\sigma$-n.e." part, these cases are covered by our case.

For $\sigma \in S_{B}$ if we put " $a^{\delta_{\sigma}}=b_{\sigma} a^{\delta_{\sigma}}$ " where $b_{\sigma}$ is the supporting projection in $B$ of $\sigma$, then $a^{\delta_{\sigma}}=a^{\varepsilon_{\sigma}} \sigma$-n.e. and $\sigma(a)=\sigma\left(a^{\delta_{\sigma}}\right)$ for all $a \in A$. The mapping $\delta_{\sigma}$ is a linear, idempotent from $A$ onto $b_{\sigma} B$ satisfying (3) and (4). That is, linear mapping $\varepsilon_{\sigma}$ and $\delta_{\sigma}$ can be considered as same one within $\sigma-n . e$. By Theorem 2, taking

$$
t_{\sigma}=b_{\sigma} t_{\varepsilon_{\sigma}}
$$

it is obvious that

$$
a^{\delta_{\sigma}}=\left(t_{\sigma} a\right)^{e} \quad \text { for every } a \in A \text { and } \sigma \in S_{B} \text {. }
$$

Under this notation, we have the following for any pair $\left(B, S_{0}\right)$ of a von

6) Let $P$ be the set of all probability measures of $(\Omega, \mathfrak{A})$. For each $\sigma \in P$ A is homomorphically represented by a von Neumann algebra $A_{\sigma}$ acting on the Hilbert space $L_{\sigma}{ }^{2}(\Omega, \mathfrak{Q})$ ( $=H_{\sigma}$ say). The algebra $A_{\sigma}$ is isomorphic to the quotient algebra $\boldsymbol{A} / \boldsymbol{N}_{\sigma}$, where $\boldsymbol{N}_{\sigma}=\left\{f \in \boldsymbol{A} ; \int|f| d \sigma=0\right\}$. The Hilbert space $H$ is defined by the direct sum $\Sigma\left\{H_{\sigma} ; \sigma \in P\right\}$ in the similar method of Takeda [11], and the von Neumann algebra $A$ is defined by the discrete direct sum $\sum\left\{A_{\sigma} ; \sigma \in P\right\}\left(=\left\{\left(\sum f_{\sigma} \mid \sigma \in P\right) ; f \in \boldsymbol{A}\right\}\right)$ where the *operation, the addition and the multiplication are introduced into $A$ by coordinatewise ones of $\left\{A_{\sigma}\right.$; $\sigma \in P\}$, that is, $\left(\sum f_{\sigma} \mid \sigma \in P\right)^{*}=\left(\sum f_{\sigma}^{*} \mid \sigma \in P\right), \quad\left(\sum f_{\sigma} \mid \sigma \in P\right)+\left(\sum g_{\sigma} \mid \sigma \in P\right)=\left(\sum(f+g)_{\sigma} \mid\right.$ $\sigma \in P$ ) etc. 
Neumann subalgebra $B$ and a set of normal states $S_{0}$ satisfying $S_{0} \subset S_{B}$ :

THEOREM 3. $B$ is sufficient for $S_{0}$ if and only if $b_{\rho} a^{\delta_{\sigma}}=b_{\sigma} a^{\delta_{\rho}}$ for every $a \in A$ and for every pair $\sigma, \rho \in S_{0}{ }^{7}{ }^{7}$

THEOREM 4. $B$ is sufficient for $S_{0}$ if and only if $B$ is sufficient for the closed convex hull $K$ of $S_{0}$ with respect to the weak topology of $A_{*}$.

Proof of Theorem 3. The "only if" part is obvious, we prove the "if" part. By the condition, for any $a \in A$

$$
a^{\delta \sigma} a^{\delta \rho}=a^{\delta} \sigma b_{\sigma} b_{\rho} a^{\delta \rho}=a^{\delta} b_{\rho} b_{\sigma} a^{\delta \rho}=a^{\delta \rho} a^{\delta_{\sigma}} .
$$

Therefore for each fixed $a \in A^{+}$, there exists $a^{\prime} \in B^{+}$such that $a^{\prime}=\sup \left\{a^{\delta_{\sigma}}\right.$; $\sigma \in S_{0}$ \} where "sup" being taken under the ordering of operators. Hence

$$
\begin{aligned}
b_{\sigma} a^{\prime}=\sup \left\{b_{\sigma} a^{\delta \rho} ; \rho \in S_{0}\right\} & =\sup \left\{b_{\rho} b_{\sigma} a^{\delta \rho} ; \rho \in S_{0}\right\} \\
& =\sup \left\{b_{\rho} a^{\delta_{\sigma}} ; \rho \in S_{0}\right\}=a^{\delta_{\sigma}}
\end{aligned}
$$

and $a^{\delta_{\sigma}}=a^{\prime} \sigma$-n.e. for each $\sigma \in S_{0}$ and $a \in A^{+}$. Since any $a \in A$ is a finite linear combination of operators in $A^{+}$and $\delta_{\sigma}$ is linear, we get $a^{\delta_{\sigma}}=a^{\prime} \sigma-n . e$. for every $a \in A$ and for some corresponding $a^{\prime} \in B$.

Proof of Theorem 4. The "if" part is obvious, we prove the "only if" part. Put $K_{0}$ the convex hull of $S_{0}$ and $K$ the weak closure of $K_{0}$ in $A_{*}$. Any $\sigma \in K_{0}$ is expressed by $\sigma=\sum_{\imath=1}^{n} \lambda_{i} \sigma_{i}$ for $\sigma_{i} \in S_{0}$ and scalars $\lambda_{i} \geqq 0\left(\sum_{i=1}^{n} \lambda_{i}=1\right)$. If $B$ is sufficient for $S_{0}$, then for each $a \in A$ there exists $a^{\prime} \in B$ such that that $a^{\delta \rho}=b_{\rho} a^{\prime}$ for every $\rho \in S_{0}$. Hence $\sigma\left(a^{\prime}\right)=\sum_{i=1}^{n} \lambda_{i} \sigma_{i}\left(a^{\prime}\right)=\sum \lambda_{i} \sigma_{i}(a)=\sigma(a)$ and $b_{\sigma} a^{\prime}=a^{\delta_{\sigma}}$. By a well-known theorem of Mazur, $K$ coincides with the closure of $K_{0}$ in the strong topology in $A_{*}$, and for any $\sigma \in K$ there exists $\left\{\sigma_{n}\right\} \subset K_{0}$ such that $\sigma_{n}(a) \rightarrow \sigma(a)$ for all $a \in A$. Therefore $\sigma(a)=\lim \sigma_{n}(a)=\lim \sigma_{n}\left(a^{\prime}\right)$ $=\sigma\left(a^{\prime}\right)$ and $a^{\delta_{\sigma}}=a^{\prime} \sigma-n . e$. then

As Lemma 1, denote $d_{\sigma}=D(\sigma / \mu)^{2}$ for $\sigma \in S_{B}$ which belongs to $L^{1}\left(B^{c}, \mu\right)$,

Lemma 2. For any $\sigma \in S_{B}, d_{\sigma}=t_{\sigma} d_{\sigma}{ }^{e}=d_{\sigma}{ }^{e} t_{\sigma}$, where $t_{\sigma}$ is defined by (12).

Proof. Since $a^{\delta_{\sigma}}=\left(t_{\sigma} a\right)^{e}$ for any $a \in A$,

$$
\mu\left(d_{\sigma} a\right)=\sigma(a)=\sigma\left(a^{\delta_{\sigma}}\right)=\mu\left(d_{\sigma} a^{\delta_{\sigma}}\right)=\mu\left(d_{\sigma}\left(t_{\sigma} a\right)^{e}\right){ }^{8)}
$$

Taking $b_{n}{ }^{\prime} \in\left(B^{c}\right)^{+}$which are commuting each other, satisfying $b_{n}{ }^{\prime} \leqq b_{n+1}{ }^{\prime}(n=1$, $2, \cdots)$ and converging to $t_{\sigma}$ in the $L_{\mu}{ }^{1}$-mean, then ${b_{n}}{ }^{e} \uparrow t_{\sigma}{ }^{e}=b_{\sigma}$ and

$$
\mu\left(d_{\sigma}{ }^{e} b_{n}{ }^{\prime}\right)=\mu\left(d_{\sigma} b_{n}{ }^{e}\right) \uparrow \mu\left(d_{\sigma} b_{\sigma}\right)=\sigma\left(b_{\sigma}\right)=1 .
$$

Since $0 \leqq d_{\sigma}^{e} b_{n}{ }^{\prime} \leqq d_{\sigma}^{e} b_{n+1}{ }^{\prime}(n=1,2, \cdots)$, it converges in the $L_{\mu}{ }^{1}$-mean to some

7) This theorem is related with the concept of the pairwise sufficiency (cf. [5]) and it will be applied to the proof of Theorem 6 .

8) If $d_{\sigma}, t_{\sigma}$ belong to $L_{\mu}{ }^{2}$, then (15) (below) is clear from (14) (cf. $\S 2$ ). 
operator in $L^{1}(A, \mu)$ and $\mu\left(d_{\sigma}{ }^{e} b_{n}{ }^{\prime}\right) \leqq 1$, and since $d_{\sigma}{ }^{e}, b_{n}{ }^{\prime}$ and $t_{\sigma}$ commute each other, by Lebesgue convergence theorem, $d_{\sigma}{ }^{e} t_{\sigma} \in L_{\mu}{ }^{1}, 0 \leqq \mu\left(d_{\sigma}{ }^{e} t_{\sigma}\right) \leqq 1$ and for every $a \in A$

$$
\mu\left(d_{\sigma}\left(t_{\sigma} a\right)^{e}\right)=\lim \mu\left(d_{\sigma}\left(b_{n}{ }^{\prime} a\right)^{e}\right)=\lim \mu\left(d_{\sigma}{ }^{e} b_{n}{ }^{\prime} a\right)=\mu\left(d_{\sigma}{ }^{e} t_{\sigma} a\right) .
$$

Combining (14) and (15), $\mu\left(d_{\sigma} a\right)=\mu\left(d_{\sigma}{ }^{e} t_{\sigma} a\right)$ and we get $d_{\sigma}=d_{\sigma}{ }^{e} t_{\sigma}=t_{\sigma} d_{\sigma}{ }^{e}$.

LEMMA 3. The B-expectation $\varepsilon_{\sigma}$ is uniquely extensible to a projection operator $p_{\sigma}$ from $L^{2}(A, \sigma)$ onto $L^{2}(B, \sigma)$ and satisfies $\left\langle a^{\varepsilon_{\sigma}} b_{1}, b_{2}\right\rangle_{\sigma}=\left\langle a b_{1}, b_{2}\right\rangle_{\sigma}$ for $b_{1}, b_{2} \in L^{2}(B, \sigma)$, where $L^{2}(B, \sigma)$ is a linear subspace of $L^{2}(A, \sigma)$ consisting of all B-measurable operators which is obviously closed. $a_{2} \in A$

Proof. Since $a^{* \varepsilon_{\sigma}} a^{\varepsilon_{\sigma}} \leqq\left(a^{*} a\right)^{\varepsilon_{\sigma}}$ for all $a \in A$ (cf. [13]), for any $a, a_{1}$ and

and

$$
\left\langle a_{1}^{\varepsilon \sigma}, a_{2}\right\rangle_{\sigma}=\sigma\left(a_{2}{ }^{*} a_{1}^{\varepsilon_{\sigma}}\right)=\sigma\left(a_{2}{ }^{\varepsilon \sigma *} a_{1}^{\varepsilon_{\sigma}}\right)=\sigma\left(a_{2}^{\varepsilon^{\varepsilon} \sigma *} a_{1}\right)=\left\langle a_{1}, a_{2}{ }^{\varepsilon \sigma}\right\rangle_{\sigma}
$$

$$
\left\langle a^{\varepsilon_{\sigma}}, a^{\varepsilon_{\sigma}}\right\rangle_{\sigma}=\sigma\left(a^{\varepsilon^{*}} a^{\varepsilon_{\sigma}}\right) \leqq \sigma\left(\left(a^{*} a\right)=\sigma\left(a^{*} a\right)=\langle a, a\rangle_{\sigma} .\right.
$$

Therefore $\varepsilon_{\sigma}$ is uniquely extended to the projection $p_{\sigma}$ from $L^{2}(A, \sigma)$ onto $L^{2}(B, \sigma)$ because the restrictions of $A$ and $B$ onto the supporting domain of $\sigma$ are dense in $L^{2}(A, \sigma)$ and $L^{2}(B, \sigma)$ respectively. The second part follows from that, $a^{\varepsilon_{\sigma}} b=p_{\sigma}(a b)$ for any $a \in A$ and $b \in B$ implies that

$$
\left\langle a^{\varepsilon \sigma} b_{1}, b_{2}\right\rangle_{\sigma}=\left\langle p_{\sigma}\left(a b_{1}\right), b_{2}\right\rangle_{\sigma}=\left\langle a b_{1}, p_{\sigma} b_{2}\right\rangle_{\sigma}=\left\langle a b_{1}, b_{2}\right\rangle_{\sigma} .
$$

Hereafter, as in Theorems 3 and 4 let $\left(B, S_{0}\right)$ be a pair of a von Neumann subalgebra $B$ and a set of normal states satisfying $S_{0} \subset S_{B}$. In the following, we prove Halmos-Savage theorem for our non-commutative case.

THEOREM 5. It is a necessary and sufficient condition for $B$ to be sufficient for $S_{0}$ that there exists a state $\pi \in S_{B}$ such that (i) $S_{0} \sim \pi$, (ii) $\pi\left(a s_{\sigma}\right.$ ) $=\pi\left(s_{\sigma} a\right)$ for each $\sigma \in S_{0}$ and for every $a \in A$ and (iii) $D(\sigma / \pi)$ coincides with a $B$-measurable operator $\sigma$-n.e. for every $\sigma \in S$.

Proof. Necessity: By the assumption, for each $a \in A$ there corresponds $a^{\prime} \in B$ such that $a^{\delta_{\sigma}}=b_{\sigma} a^{\prime}$, and by Theorem 2 there exists $t_{\sigma} \in L^{1}\left(B^{c}, \mu\right)$ such that $a^{\delta \sigma}=\left(t_{\sigma} a\right)^{e}$ for all $a \in A$ and $t_{\sigma}{ }^{e}=b_{\sigma}$, where the notations $t_{\sigma}$ and $\delta_{\sigma}$ refer to the equations (12) and (13). Whence for each pair $\sigma, \rho \in S_{0}$

$$
\begin{aligned}
\mu\left(b_{\rho} t_{\sigma} a\right)=\mu\left(b_{\rho}\left(t_{\sigma} a\right)^{e}\right) & =\mu\left(b_{\rho} a^{\delta \sigma}\right)=\mu\left(b_{\rho} b_{\sigma} a^{\prime}\right) \\
& =\mu\left(b_{\sigma} b_{\rho} a^{\prime}\right)=\mu\left(b_{\sigma} a^{\delta \rho}\right)=\mu\left(b_{\sigma}\left(t_{\rho} a\right)^{e}\right)=\mu\left(b_{\sigma} t_{\rho} a\right)
\end{aligned}
$$

and $b_{\rho} t_{\sigma}=b_{\sigma} t_{\rho}$. Since $b_{\sigma} t_{\sigma}=t_{\sigma}$ and $b_{\rho} t_{\rho}=t_{\rho}$,

$$
t_{\sigma} t_{\rho}=b_{\sigma} t_{\sigma} b_{\rho} t_{\rho}=b_{\rho} t_{\sigma} b_{\sigma} t_{\rho}=t_{\rho} t_{\sigma} .
$$

While for any $b^{\prime}, b^{\prime \prime} \in B^{c}$ and $b \in B, \mu\left(b^{\prime e} b^{\prime \prime e} b\right)=\mu\left(b^{\prime} b^{\prime \prime e} b\right)=\mu\left(b^{\prime \prime e} b^{\prime e} b\right)$ and $b^{\prime e} b^{\prime \prime e}$ $=b^{\prime \prime e} b^{\prime e}$. Since $d_{\sigma}, d_{\rho} \in L^{1}\left(B^{c}, \mu\right), d_{\sigma}$ and $d_{\rho}$ are approximated by sequences in $B^{c}$ respectively, and we have $d_{\sigma}{ }^{e} d_{\rho}{ }^{e}=d_{\rho}{ }^{e} d_{\sigma}{ }^{e}$. Since by Lemma $2 d_{\sigma}=t_{\sigma} d_{\sigma}{ }^{e}$ 
and $d_{\rho}=t_{\rho} d_{\rho}{ }^{e}$,

$$
d_{\sigma} d_{\rho}=t_{\sigma} d_{\sigma}{ }^{e} t_{\rho} d_{\rho}{ }^{e}=t_{\sigma} t_{\rho} d_{\sigma}{ }^{e} d_{\rho}{ }^{e}=t_{\rho} t_{\sigma} d_{\rho}{ }^{e} d_{\sigma}{ }^{e}=t_{\rho} d_{\rho}{ }^{e} t_{\sigma} d_{\sigma}{ }^{e}=d_{\rho} d_{\rho} .
$$

Let $d_{\sigma}=\int \lambda d E_{\lambda}(\sigma)$ be the spectral resolution of $d_{\sigma}\left(\sigma \in S_{0}\right)$ and $C$ the von Neumann subalgebra generated by $\left\{E_{\lambda}(\sigma) ; \lambda \geqq 0, \sigma \in S_{0}\right\}$, then $C$ is commutative and $d_{\sigma}\left(\sigma \in S_{0}\right)$ are $C$-measurable, moreover there exists a projection $p_{0} \in C$ such that $p_{0}=\sup \left\{s_{\sigma} ; \sigma \in S_{0}\right\}$. Let $F$ be a family of all projections $p \in C$ such that $p \in F$ if and only if $p \leqq s_{\sigma}$ for some $\sigma \in S_{0}$. By the countable decomposability of $C$ and by Zorn lemma there exist a countable $\left\{\sigma_{n}\right\} \subset S_{0}$ and $\left\{p_{n}\right\} \subset F$ such that $p_{n} \leqq s_{\sigma_{n}}$ and $\sup _{n=1}^{\infty} p_{n}=p_{0}$. Putting $\pi=\sum_{n=1}^{\infty} \sigma_{n} / 2^{n}, \pi$ belongs to the closed convex hull of $S_{0}$ (which is contained in $S_{B}$ ) and $p_{0}=s_{\pi}$, and hence $\pi \sim S_{0}$. Consequently by Theorem $4 a^{\delta \pi}=b_{\pi} a^{\prime}$ ( $a^{\prime}$ is the operator in $B$, cf. Def. 2) and by $b_{\sigma} \leqq b_{\pi}$ we get $b_{\sigma} t_{\pi}=t_{\sigma}$ and $^{9)}$

$$
d_{\sigma} d_{\pi^{-1}}=\left(t_{\sigma} d_{\sigma}{ }^{e}\right)\left(t_{\pi} d_{\pi^{e}}\right)^{-1}=t_{\sigma} t_{\pi^{-1}} d_{\sigma}{ }^{e} d_{\pi^{e-1}}=s_{\sigma} b_{\sigma} d_{\sigma}{ }^{e} d_{\pi^{e-1}}=s_{\sigma} d_{\sigma}{ }^{e} d_{\pi^{e-1}}
$$

and $d_{\sigma} d_{\pi^{-1}}=d_{\sigma}{ }^{e} d_{\pi^{e-1}} \sigma-n . e$. Since $d_{\sigma}$ commutes with $d_{\pi}$ and $d_{\mu}(=1), D(\sigma / \pi) \geq 0$ and $d_{\sigma} d_{\pi^{-1}}=D(\sigma / \pi)^{2}$. Therefore $D(\sigma / \pi)$ coincides with the $B$-measurable operator $\left(d_{\sigma}^{e} d_{\pi^{e-1}}\right)^{1 / 2} \sigma-n . e$. The condition (ii) is obvious from (16) and the construction of $\pi$.

Sufficiency: For any fixed $\sigma \in S_{0}$, put $A_{\sigma}=\left\{s_{\sigma} a s_{\sigma} ; a \in A\right\}$ and $B_{\sigma}=\left\{s_{\sigma} b s_{\sigma}\right.$; $b \in B\}$. Then $A_{\sigma}$ and $B_{\sigma}$ are von Neumann algebras (acting on $s_{\sigma} H$ ) with the parallel properties (in $\S 2$ ) of $A$ and $B$ respectively. Since for any $a \in A$, $b \in B$ and $\rho \in S_{B}$

$$
\rho\left(s_{\sigma} b s_{\sigma} a s_{\sigma}\right)=\rho\left(b s_{\sigma} a s_{\sigma}\right)=\rho\left(s_{\sigma} a s_{\sigma} b\right)=\rho\left(s_{\sigma} a s_{\sigma} b s_{\sigma}\right),
$$

putting $\pi^{\prime}(a)=\pi(a) / \pi\left(s_{\sigma}\right)$ and $\sigma^{\prime}(a)=\sigma(a)$ for $a \in A_{\sigma}, \pi^{\prime}$ and $\sigma^{\prime}$ belong to $S_{B \sigma}$ as states of $A_{\sigma}$. By the assumptions (ii) and (iii), $D\left(\sigma^{\prime} / \pi^{\prime}\right)$ belongs to $L^{2}\left(B_{\sigma}, \pi^{\prime}\right)$, and by Lemma 3 (for $A_{\sigma}, B_{\sigma}$ and $\pi^{\prime}$ ) we get immediately

$$
\begin{aligned}
\sigma\left(\left(s_{\sigma} a s_{\sigma}\right)^{\varepsilon_{\pi}} b\right) & =\left\langle\left(s_{\sigma} a s_{\sigma}\right)^{\varepsilon} \pi b D(\sigma / \pi), D(\sigma / \pi)\right\rangle_{\pi} \\
& =\left\langle s_{\sigma} a s_{\sigma} b D(\sigma / \pi), D(\sigma / \pi)\right\rangle_{\pi}=\sigma\left(s_{\sigma} a b s_{\sigma}\right)=\sigma(a b)=\sigma\left(a^{\varepsilon_{\sigma}} b\right)
\end{aligned}
$$

and $b_{\sigma}\left(s_{\sigma} a s_{\sigma}\right)^{\varepsilon_{\pi}}=b_{\sigma} a^{\varepsilon_{\sigma}}=a^{\delta_{\sigma}}$ for every $a \in A$. While the condition (ii) implies that $\pi\left(s_{\sigma} a b\right)=\pi\left(a b s_{\sigma}\right)=\pi\left(a s_{\sigma} b\right)$ for every $b \in B$ and $\left(s_{\sigma} a\right)^{\delta \pi}=\left(a s_{\sigma}\right)^{\delta_{\pi}}$ for every $a \in A$. Therefore again by (ii), for $a \in A^{+}, b \in B^{+}$and $b_{1}=b^{1 / 2}$

and

$$
\pi\left(s_{\sigma} a b\right)=\pi\left(s_{\sigma} b_{1} a b_{1}\right)=\pi\left(s_{\sigma} b_{1} a b_{1} s_{\sigma}\right) \leqq \pi\left(b_{1} a b_{1}\right)=\pi(a b)
$$

$$
a^{\delta_{\sigma}}=b_{\sigma} a^{\varepsilon_{\sigma}}=b_{\sigma}\left(s_{\sigma} a s_{\sigma}\right)^{\varepsilon_{\pi}} \leqq b_{\pi}\left(s_{\sigma} a s_{\sigma}\right)^{\varepsilon_{\pi}}=\left(s_{\sigma} a s_{\sigma}\right)^{\delta_{\pi}}=\left(s_{\sigma} a\right)^{\delta_{\pi}} \leqq a^{\delta_{\pi}}
$$

Put $f(a)=\sigma\left(a^{\delta} \pi\right)-\sigma\left(a^{\delta \sigma}\right)$. Then $f$ is a positive linear functional of $A$ and

$$
f(1)=\sigma\left(1^{\delta_{\pi}}\right)-\sigma\left(1^{\delta \sigma}\right)=\sigma\left(b_{\pi}\right)-\sigma\left(b_{\sigma}\right)=\sigma(1)-\sigma(1)=0 .
$$

Consequently $f=0$ and $\sigma\left(a^{\varepsilon} \pi\right)=\sigma\left(a^{\delta \pi}\right)=\sigma\left(a^{\delta \sigma}\right)=\sigma\left(a^{\varepsilon_{\sigma}}\right)$. Thus we obtain $a^{\varepsilon_{\sigma}}=a^{\varepsilon_{\pi}}$

9) For any positive and measurable operator $t, t^{-1}$ is defined such as the closure of the inverse operator in its supporting manifold and zero in its orthocomplimented manifold, which is also positive and measurable. 
$\sigma$-n.e. for every $a \in A$. Since $\sigma$ is arbitary in $S_{0}, B$ is sufficient for $S_{0}$.

REMARK 3. In the original case of Theorem 5 (cf. Theorem 1 of [5]), the countable decomposability has not been assumed, but it has been discussed for a set of probability measures dominated by a probability measure $\bar{p}$, which implies that the multiplication algebra of all bounded random variables defined $\bar{p}$-n.e. becomes a countably decomposable von Neumann algebra, and it reduces to our case.

In the rest part of this section, we shall describe for our case a related concept of a theorem of Halmos-Savage with respect to the pairwise suffciency in probability theory. The following will be obtained immediately from Theorem 5:

COORLLARY 5.1. If the set $S_{0}$ consists of the faithful normal states, then it is a necessary and sufficient condition for $B$ to be sufficient for $S_{0}$ that $D(\sigma / \rho)$ is $B$-measurable for every pair of states $\sigma, \rho \in S_{0}$.

Definition 3. A closed linear subspace $\boldsymbol{C}$ of $A_{*}$ is called abelien subspace if $C$ is generated by $\left\{l_{c} \tau ; c \in C\right\}$ for a certain commutative, weakly closed self-adjoint subalgebra $C$ of $A$ and for a certain normal trace $\tau$ of $A$, where the operation $l_{c}$ is defined by $\left(l_{c} f\right)(a)=f(c a)\left(f \in A^{*}\right)$.

For example, the closed linear subspaces of $A_{*}$ generated by the set of all normal traces of $\boldsymbol{A}$ is an abelian subspace. In general, the real part of any abelian subspace $C$ of $A_{*}$ is an abstract $(L)$-space in the sense of Kakutani (cf. [6]) where the ordering in $C$ is defined from the positive definiteness. ${ }^{10)}$ By Radon-Nikodym theorem due to Dye, we can easily see that:

Lemma 4. If a closed linear and self-adjoint subspace $C$ of $A_{*}$ is an abelian subspace, then $\boldsymbol{C} \frown A_{*}{ }^{+}$generates $\boldsymbol{C}$ and $d_{\sigma}, d_{\rho}$ commute each other for any pair of normal states $\sigma, \rho$ in $\boldsymbol{C}$.

Denote for any pair of normal states $\sigma, \rho D(\sigma, \rho)=D(\sigma /(\sigma+\rho) / 2)$. Under these terminologies we prove the following:

THEOREM 6. It is a necessary and sufficient condition for $B$ to be sufficient for $S_{0}$ that $\left(1^{\circ}\right) S_{0}$ is contained in an abelian subspace of $A_{*}$ and $\left(2^{\circ}\right) D(\sigma, \rho)$ coincides with a B-measurable operator $\sigma$-n.e. for every pair $\sigma, \rho \in S_{0}$.

Proof. Necessity: We refer to the proof of Theorem 5. Let $C$ be the von Neumann subalgebra and $\pi$ the normal state in $S_{B}$ which were defined in the part of the necessity of that proof. Then $C$ is commutative, $d_{\sigma} \in L^{1}(C, \mu)$ for every $\sigma \in S_{0}$ and further by the construction of $\pi d_{\pi} \in L^{1}(C, \mu)$. Putting

10) We shall give a characterization of the abelian subspace under the terminology of abstract $(L)$-space in another occasion. 
$\boldsymbol{C}=\left\{f_{x} ; x \in L^{1}(C, \mu)\right\}$ (where $f_{x}(a)=\mu(x a)$ ), $C$ is obviously an abelian subspace of $A_{*}$ and contains $S_{0}$. Consequently the closed convex hull $K$ of $S_{0}$ is contained in $C$ and for any pair $\sigma, \rho \in K$ with $\sigma \prec \rho D(\sigma / \rho) \geqq 0$, and by Theorem 4 and the proof of Theorem $5 d_{\sigma} d_{\pi^{-1}}=D(\sigma / \pi)^{2}=s_{\sigma} v_{\sigma}$ for certain $B$-measurable positive operator $v_{\sigma}$, which depends on $\sigma$, for each $\sigma \in K$. Hence

$$
d_{\sigma} d_{\rho^{-1}}=s_{\sigma}\left(v_{\sigma} d_{\pi}\right)\left(v_{\rho} d_{\pi}\right)^{-1}=s_{\sigma}\left(v_{\sigma} v_{\rho}^{-1}\right) s_{\pi}=s_{\sigma} v_{\sigma} v_{\rho}^{-1} \text {. }
$$

Since $v_{\sigma}$ and $v_{\rho}$ can be taken to be positive and commuting each other, $D(\sigma / \rho)$ $=\left(d_{\sigma} d_{\rho^{-1}}\right)^{1 / 2}=s_{\sigma}\left(v_{\sigma} v_{\rho^{-1}}\right)^{1 / 2}$ and $\left(2^{\circ}\right)$ follows immediately from this.

Sufficiency: By the condition $\left(1^{\circ}\right)$ and by Lemma $4, d_{\sigma} d_{\rho}=d_{\rho} d_{\sigma}$ for each pair $\sigma, \rho \in S_{0}$ and $d_{\sigma} d_{\tau}=d_{\tau} d_{\sigma}$ (where $\tau=(\sigma+\rho) / 2$ ), and further $\tau\left(\alpha s_{\sigma}\right)=\tau\left(s_{\sigma} a\right)$ for every $a \in A$. Therefore by the same manner of the proof of Theorem 5, $a^{\varepsilon_{\sigma}}=a^{\varepsilon_{\tau}} \sigma$-n.e. and similarily $a^{\varepsilon \rho}=a^{\varepsilon_{\tau}} \rho$-n.e.. Consequently $a^{\delta_{\sigma}}=b_{\sigma} a^{\varepsilon_{\sigma}}=b_{\sigma} a^{\varepsilon_{\tau}}$, $a^{\delta \rho}=b_{\rho} a^{\varepsilon_{\tau}}$ and

$$
b_{\rho} a^{\delta_{\sigma}}=b_{\rho} b_{\sigma} a^{\delta \tau}=b_{\sigma} b_{\rho} a^{\varepsilon_{\tau}}=b_{\sigma} a^{\delta \rho} .
$$

Thus by Theorem 3 we get the sufficiency of $B$ for $S_{0}$.

\section{Uniqueness of expectation.}

In this final section, we shall give a simple application of the sufficiency of a von Neumann subalgebra to the uniqueness of expectation on it. This is essential for non-commutative case.

THEOREM 7. The following conditions are equivalent each other:

(i) $B$ is sufficient for $S_{B}$,

(ii) $B^{c} \subset B$, where $B^{c}=B^{\prime} \frown A$,

(iii) The B-expectation is uuique.

Proof. (i) $\rightarrow$ (ii): For $b^{\prime} \in\left(B^{c}\right)^{+}$, put $b=\left(b^{\prime}+1\right) / \mu\left(b^{\prime}+1\right)$ and $\sigma(a)=\mu(b a)$ $(a \in A)$. Then $\sigma \in S_{B}$ and is 'faithful. Hence $\varepsilon_{\sigma}=\varepsilon_{\mu}(=e)$, and $a^{\delta_{\sigma}}=b_{\sigma} a^{\varepsilon_{\sigma}}=a^{\varepsilon_{\sigma}}$ by $b_{\sigma}=1$. By Theorem 2, taking $t_{\sigma}$ such as (12), then for all $a \in A$

$$
\mu\left(t_{\sigma} a\right)=\mu\left(\left(t_{\sigma} a\right)^{e}\right)=\mu\left(a^{\varepsilon} \sigma\right)=\mu\left(a^{e}\right)=\mu(a)
$$

which implies $t_{\sigma}=1$. While $b=d_{\sigma}=t_{\sigma} d_{\sigma}{ }^{e}$ (by Lemma 2) $=d_{\sigma}{ }^{e}$ and $b \in B$. Therefore $b^{\prime} \in B$, and $B^{c} \subset B$, because each operator in $B^{c}$ is expressed by a finite linear combination of the operators in $\left(B^{c}\right)^{+}$.

(ii) $\rightarrow$ (iii): Let $\varepsilon$ be a $B$-expectation. Putting $\sigma(a)=\mu\left(a^{\varepsilon}\right)$, then $\sigma \in S_{B}$ and $\varepsilon_{\sigma}=\varepsilon$. Since $a^{\varepsilon}=\left(t_{\sigma} a\right)^{e}$ for $t_{\sigma} \in L^{1}\left(B^{c}, \mu\right)$ with $t_{\sigma}{ }^{e}=1$ and by (ii) $t_{\sigma}$ belongs to $L^{1}(B, \mu)$, we obtain $t_{\sigma}=1$ and $a_{\varepsilon}=\left(t_{\sigma} a\right)^{e}=a^{e}$.

(iii) $\rightarrow$ (i ) follows immediately from Theorem 1 .

COROLlaRy 7.1. If $B=N^{c}$ for certain commutative von Neumann subalgebra $N$, then the B-expectation is unique.

Proof. $N \subset N^{\prime}$ and $N \subset A$ imply $N \subset N^{c}=B$ and $B^{\prime} \subset N^{\prime}$. Hence $B^{c} \subset N^{\prime} \frown A$ $=N^{c}=B$ and we get the proof by Theorem 7 . 
From this corollary, Theorem 2 in [9] (which will be stated in Cor. 7.1') follows such as: For a projection $p \in A$, denote $C_{p}=\{p\}^{\prime} \frown A$ and $a^{\mid p}=p a p$ $+(1-p) a(1-p)$ for every $a \in A, \mid p$ being a notation of von Neumann (e.g. see [9]). Then $\mid p$ is obviously a $C_{p}$-expectation and by Corollary 7.1 every $C_{p}$-expectation is unique and hence is represented by $\mid p$. Moreover let $\{p, q, \cdots, r, \cdots\}$ be a countable family of projections in $A$ which are commuting each other, and put $B=C_{p} \frown C_{q} \frown \cdots$. Then $B=\{p, q, \cdots\}^{c}$, and the $B$-expectation is unique. While for every $a \in A a^{|p| g|\cdots| r}$ converges in the strong operator topology to an operator $b \in B$ (cf. [9], [14]) and denote $a^{|p| q \mid \cdots}=b$. Then the mapping $|p| q \mid \cdots$ is a $B$-expectation, which has been called the von Neumann's operation defined by the von Neumann subalgebra $\{p, q, \cdots\}^{\prime \prime}$ (cf. [9]). Consequently for the subalgebra $B$, every $B$ expectation is represented by the von Neumann's operation $|p| q \mid \cdots$. Especially, if the Hilbert space $H$, on which $A$ acts, is separable, and if $N$ is commutative, then $N$ is generated by a countable family of projections in it, therefore we obtain

CoRollary 7.1'. For the von Neumann subalgebra $B\left(=N^{c}\right)$, the B-expectation coincides with the von Neumann's operation defined by $N$, that is, for any countable family of projections $p, q, \cdots$ which generates $N,|p| q \mid \cdots$ defines the unique $B$-expectation and hence is determined only by $N$.

This corollary contains a von Neumann proposition, which has been proved in [9], for our special von Neumann algebra $A$. Concerning the von Neumann's operation, we shall give a further remark (cf. Cor. $7 \cdot 1^{\prime \prime}$ ). For any von Neumann subalgebra $M, M^{c}$ satisfies $M^{c}=M^{c c c}\left(=\left(\left(M^{c}\right)^{c}\right)^{c}\right)$. Indeed, $a \in M^{c}$ is equivalent to that $a a^{\prime}=a^{\prime} a$ for all $a^{\prime} \in M^{c^{\prime}}=M \smile A^{\prime}$ which is also equivalent to $a \in\left(M^{c} \smile A^{\prime}\right) \frown A\left(=M^{c c c}\right)$, where $M \smile A^{\prime}$ means the smallest von Neumann subalgebra containing $M$ and $A^{\prime}$. Furthermere if $M$ is commutative, then $M^{c c}$ is also commutative. This is clear by that $M^{c c}=\left(M^{\prime} \frown A\right)^{\prime}$ $\frown A=\left(M \cup A^{\prime}\right) \frown A$. We take $N$, and the Hilbert space $H$ as in the Cor. 7.1', then $N^{c c c}=N^{c}=B, N^{c c}$ is commutative and is generated by a countable family of projections $p, q, \cdots$. Therefore the von Neumann's operation defined by $N^{c c}$ is also $B$-expectation, that is, we obtain

Corollary 7.1". The von Neuman's operations defined by $N$ and $N^{c c}$ are identical.

As a special case of Cor. 7.1 and Cor. $7.1^{\prime}$, we have the following:

COROLlary 7.2. If $B$ is a maximally abelian subalgebra, then the $B$ expectation is unique, and furthermore if the Hilbert space $H$ is separable then it is a von Neumann's operation defined by $B$.

If the converse of the last part of this corollary holds, then $B$ is maximally abelian, that is, for commutative $B$, the equality "the B-expectation 
$=$ the von Neumann's operation defined by $B$ " is a characteristic property of the maximality of $B$ (cf. Theorem 3 of [9]).

Let $T$ be the set of all normal traces of $A$, then from the proof of Theorem 7 we have:

CoRollary 7.3. For a von Neumann subalgebra $B, B$ is sufficient for $T$ if and only if $A^{\prime} \frown A \subset B$.

This contains a result of Dixmier (cf. Proposition 8 of [3]) for the present restricted algebra $A$.

\section{REFERENCES}

[1] Bahadur, R. R., Sufficiency and statistical decision function. Ann. Math. Statist. 25 (1954), 423-462.

[2] Dixmier, J., Des algèbres d'opérateurs dans l'espace Hilbertien. 1957, Paris.

[3] Dixmier, J., Formes linéaires sur un anneau d'opérateurs. Bull. Soc. Math. France 81 (1953), 9-39.

[4] Dye, H. A., The Radon-Nikodym theorem for finite rings of operators. Trans. Amer. Math. Soc. 72 (1952), 243-280.

[5] Halmos, P. R., AND L. J. Savage, Application of the Radon-Nikodym theorem to the theory of sufficient statistics. Ann. Math. Statist. 20 (1949), 225-241.

[6.] KAKUTANI, S., Concrete representation of abstract $(L)$-spaces and the mean ergodic theorem. Ann. Math. 42 (1941), 523-537.

[7] Nakamura, M., On the direct product of finite factors. Tôhoku Math. J. 6 (1954), 205-209.

[8] Nakamura, M., AND T. Turumaru, Expectations in an operator algebra. Tôhoku Math. J. 6 (1954), 182-188.

[9] Nakamura, M. AND H. Umegaki, On a proposition of von Neumann. Kōdai Math. Sem. Rep. 8 (1956), 142-144.

[10] SegAL, I. E., A non-commutative extension of abstract integration. Ann. Math. 57 (1953), 401-457.

[11] TAKedA, Z., Conjugate spaces of operator algebras. Proc. Jap. Acad. 30 (1954), 90-95.

[12] TAKesaki, M., On the direct product of $W^{*}$-factor. Tôhoku Math. J. 10 (1958), 116-119.

[13] Tomiyama, J., On the projection of norm one in $W^{*}$-algebra. Proc. Jap. Acad. 33 (1957), 608-612.

[14] Umegaki, H., Conditional expectation in an operator algebra. Tôhoku Math. J. 6 (1954), 177-181.

[15] Umegaki, H., Conditional expectation in an operator algebra, II. Tôhoku Math. J. 8 (1956), 86-100.

Department of Mathematics, Tokyo Intitute of TechNology. 